\title{
The vine; an emblematic plant bio-indicatrice of the climatic variations, presentation of project ADVICLIM / La vigne ; une plante bio-indicatrice emblématique des variations climatiques, présentation du projet ADVICLIM
}

\author{
Joël Rochard \\ IFV (Institut Français de la Vigne et du Vin), 17 rue Jean Chandon Moët, 51200 Epernay, France
}

\begin{abstract}
The probable evolution of the climate, grows rich by a historical wine glance. The vine could illuminate this major challenges linked to climate change in particular thanks to the historical analysis of "ban des vendanges" de (grape harvest data) as well as the physiological and phenologic observations in the various wine-producing areas, the last and future evolution of the climate. The wine historians and experts studied the variability of the round of applause of vintage during the last five centuries. The precocity observed since the end of 1980 is from 10 to 20 days comparatively in the middle of the 20th century. In complement the recent historical data make it possible to apprehend in a simple way the influence of the climate change on the dates of grape harvest (10 days average precocity per additional degree Celsius). An European LIFE project ADVICLIM (www.adviclim.eu), coordinated by CNRS aims to set up a network of international measurements and a Web platform which will make it possible to the wine growers to evaluate the impacts of the climate change on their pieces. The objective of the communication is to present the historical aspects of the dates of grape harvest in link with the variations of the climate and to determine the future trends to be planned to adapt to the various scenarios during the 21 st century.
\end{abstract}

\begin{abstract}
Résumé. L'évolution probable du climat, s'enrichit d'un regard viticole historique. La vigne pourrait éclairer cet enjeux majeur lié aux changements climatiques, notamment grâce à l'analyse historique du ban des vendanges ainsi que les observations physiologiques et phénologiques dans les différentes régions viticoles, l'évolution passée et future du climat. Les historiens et les experts viticoles ont étudié la variabilité du ban des vendanges au cours des cinq derniers siècles. La précocité observée depuis la fin des années 1980 est de 10 à 20 jours comparativement au milieu du XXe siècle. En complément les données historiques récentes permettent d'appréhender de manière simple l'influence du changement climatique sur les dates des vendanges (précocité moyenne de 10 jours par degré Celsius supplémentaire). Un projet européen LIFE ADVICLIM (www.adviclim.eu), coordonné par le CNRS a pour objectif de mettre en place un réseau de mesures internationales et une plate-forme Web qui permettront aux viticulteurs d'évaluer les impacts du changement climatique sur leurs parcelles. L'objectif de la communication est de présenter les aspects historiques des dates des vendanges en lien avec les variations du climat et de cerner les évolutions futures à envisager pour s'adapter aux différents scénarios au cours du XXIe siècle.
\end{abstract}

\section{Introduction}

La France a accueilli du 30 novembre au 11 décembre 2015 la 21ème conférence sur le Climat (COP21). Elle a permis un premier accord universel et contraignant sur le climat pour maintenir la température globale en deçà de $2{ }^{\circ} \mathrm{C}$. Dans ce cadre, il est intéressant de souligner que la vigne apporte un éclairage historique sur cet enjeu majeur lié aux changements climatiques (Fig. 1).

Comme le souligne I. Garcia de Cortazar Atauri « La température est le facteur le plus important dans la date de manifestation des étapes de développement de la flore »... «La base de données la plus ancienne dont la communauté scientifique dispose remonte au IXe siècle. Il s'agit de la date de floraison des cerisiers au Japon, phénomène qui là-bas est un véritable événement national.
En France, on peut remonter jusqu'au XIVe siècle en utilisant les dates des vendanges. Elles ne constituent pas un stade phénologique en soi puisqu'elles sont décidées par l'homme mais donnent une idée de la maturation du fruit $\gg$.

Ainsi le ban des vendanges déterminé par les seigneurs, les moines puis après la révolution française au niveau communal puis au cours du XXe siècle par les décrets d'appellation d'origine, constituent comme le précise V. Daux «des archives continentales d'un grand intérêt du fait de leur résolution annuelle, de leur datation précise et des relations fortes et statistiquement significatives qui les lient à la température moyenne de la période de maturation $d u$ raisin $\gg$.

En complément, par l'approche culturelle liée au vin, les vendanges font toujours l'objet d'attentions 

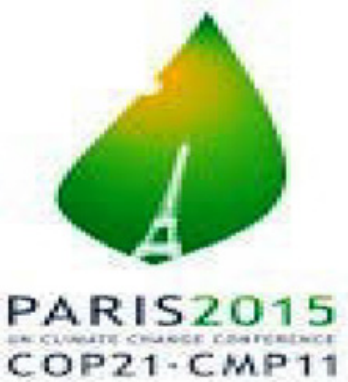

Figure 1. Logo COP21.

médiatiques et le lien entre la vigne, le climat et son évolution est souvent développé dans les médias «grand public $\gg[1]$.

\section{Evolution du climat : une constance de l'histoire de la terre}

Depuis l'apparition de la vie sur terre, le climat n'a cessé d'évoluer. La modification du niveau de la mer associée à la présence des fossiles, la disparition de certaines espèces, sont autant de témoins des changements climatiques liés aux cycles naturels (facteurs astronomiques) ou à des événements exceptionnels (météorites, activités volcanique etc.). Les traces géologiques de cette évolution (sédiment, alluvions, etc.) sont parfois des composantes de la spécificité de certains terroirs et de la richesse des paysages viticoles [2].

Parallèlement à l'évolution naturelle, il semble que les activités humaines et industrielles conduisent à une accélération rapide des modifications du climat et notamment du réchauffement. L'analyse des relevés de température par Météo France démontre une tendance au réchauffement de 0,8 à $1.5^{\circ} \mathrm{C}$ selon les régions françaises depuis un siècle, avec une accélération au cours des trois dernières décennies. Par ailleurs, une tendance à la diminution du nombre de jours de gelées et à l'augmentation du nombre de jours de forte chaleur a pu être décelée au cours du siècle précédent.

Le développement durable met en lumière l'effet de l'activité des hommes à long terme sur la planète. L'évolution probable du climat, soumis à de nombreuses incertitudes, s'enrichit d'un regard viticole historique. Si le vin a été l'initiateur dans la compréhension scientifique de la microbiologie, grâce aux travaux de Pasteur, la vigne pourrait bien éclairer, notamment grâce à l'analyse historique du ban des vendanges ainsi que les observations physiologiques et phénologiques dans les différentes régions viticoles, l'évolution passée et future du climat (Fig. 2) [3].

Un bio-indicateur désigne des espèces biologiques ou animales qui, du fait de leurs particularités écologiques, constituent l'indice précoce de modifications biotiques ou abiotiques de l'environnement dues à des activités humaines à l'origine de la majeure partie du réchauffement des cinquante dernières années.

\subsection{Climat et religion}

Les fléaux climatiques étaient souvent associés à des punitions divines. Ainsi, J. Berlioz, souligne que le diable et les démons sont considérés dans un contexte populaire

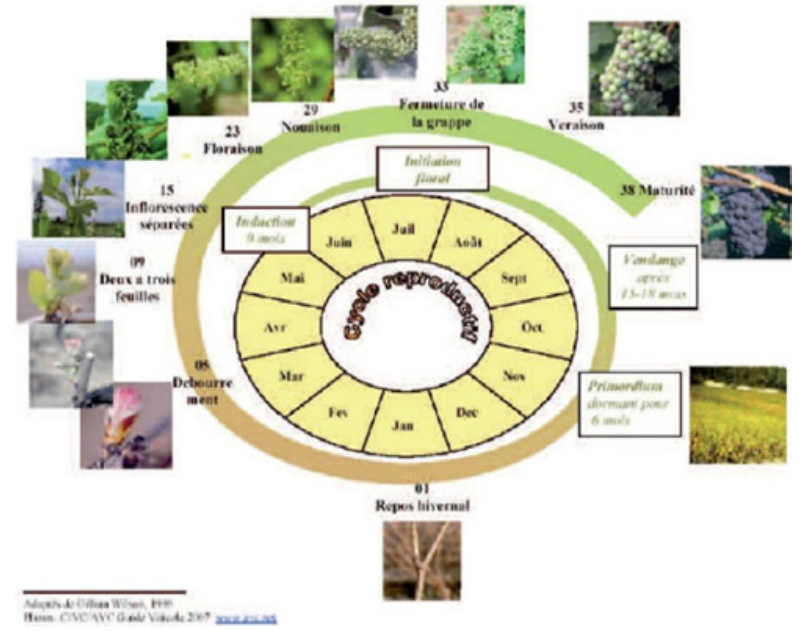

Figure 2. Cycle reproductif de la vigne d'après M. Greven et J. Rochard.

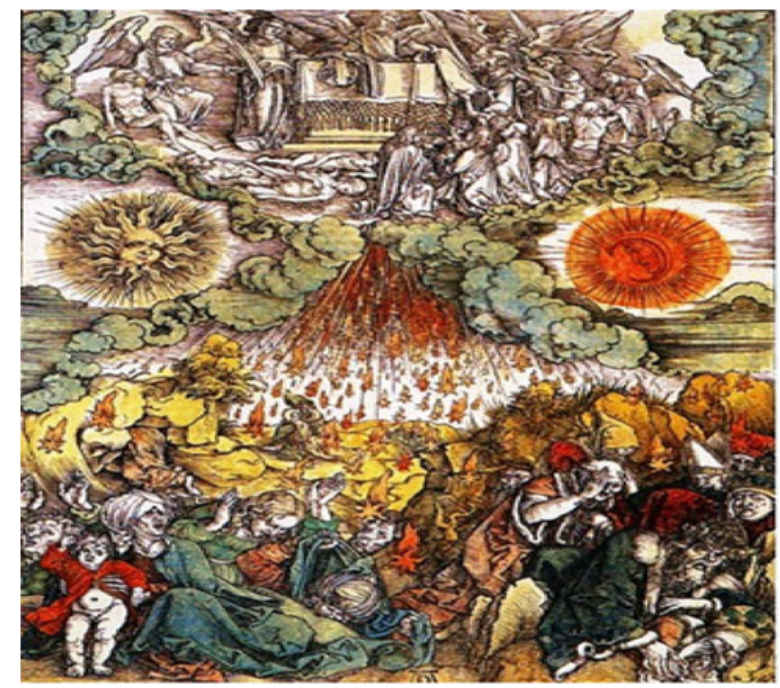

Figure 3. L'Apocalypse de Jean met en scène un terrifiant bouleversement climatique «Et les astres du ciel s'abattirent sur la terre » (Albrecht Dürer, Venise, Musée Correr ; cl. Dagli Orti). Source : J. Berlioz, la foudre, colère de Dieu, revue l'histoire $\mathrm{n}^{\circ}$ 257, septembre 2001.

comme des sources de calamités. Pour conjurer ces fléaux, il n'était pas rare de faire appel à des pratiques magiques, relayées par les superstitions. Pour l'église, cette peur des aléas climatiques était l'occasion de sacraliser les prières, psaumes, litanies, oraison afin d'éloigner les calamités. Le recours aux saints (Saint-Vincent par exemple) s'inscrit également dans une approche culturelle en lien avec les divinités (Fig. 3).

\subsection{Climat et Viticulture}

Née probablement dans la région du Caucase, à proximité de la Mer Noire, la vigne cultivée n'a cessé de conquérir de nouvelles régions (Moyen Orient, Bassin méditerranéen), au cours de l'expansion de la civilisation grecque puis romaine. Plus récemment, le développement du transport par bateau, à l'origine des colonisations a permis une implantation des vignobles dans les pays du nouveau monde (Amérique du Nord, Hémisphère sud, Asie). 
Si la vigne est dotée d'une capacité d'adaptation exceptionnelle, et si l'homme sait inventer les techniques culturales dans les conditions les plus extrêmes, cette plante liane n'a trouvé majoritairement son terrain de prédilection que dans quelques zones du globe correspondant à des conditions climatiques spécifiques (20 à $53^{\circ}$ de latitude dans l'hémisphère Nord et 20 à $42^{\circ}$ dans l'hémisphère sud) (Fig. 4).

Au-delà de la répartition mondiale, à un niveau plus local, l'optimum qualitatif d'un terroir est lié à un savoirfaire du vigneron (mode de culture, cépages...), qui sait tirer profit des spécificités microclimatiques de sa région [4].

Les particularités climatiques de l'année conditionnent le site reproductif de la vigne. Le débourrement selon Baggiolini, correspond à la date à laquelle $50 \%$ des bourgeons sont au stade $\mathrm{C}$, pointe verte visible, la floraison est définie par la date à laquelle $50 \%$ des fleurs sont ouvertes. La véraison est le moment où les baies entrent dans la phase finale de maturation [5].

\section{Influence du climat sur la vigne [6].}

d'après Oz Clarke, extrait de « Atlas Hachette des vins du Monde», 1995.

Sans l'influence du Gulf Stream, qui réchauffe les côtes occidentales de la France, les crus classés du Médoc n'existeraient pas. Sans les brouillards qui refroidissent les rivages californiens, nous ne pourrions apprécier les vins de Cabernet de la Napa Valley. Sans le courant de Benguela, qui naît dans l'antarctique et rafraîchit le front fiévreux du Cap, la canicule sud-africaine rendrait impossible l'élaboration de vins agréables. Les barrières naturelles qui protègent l'Alsace, l'état de Washington, aux Etats-Unis, et Marlborough, en Nouvelle-Zélande, assurent un automne long et sec, durant lequel les fruits ont tout le temps de parvenir à maturité. Sans l'influence adoucissante des lacs Eriée et Ontario à la frontière américano-canadienne, il n'y aurait pas de viticulture en Ontario, et rien ne pourrait mûrir au nord de l'état de New-York ou dans l'Ohio.

Inversement, on ne peut que déplorer les gelées tardives qui apparaissent par une belle nuit d'avril et déciment la vendange à Chablis ; la sécheresse qui s'installe sournoisement en Nouvelle-Galles du sud, en Australie met les vignes les plus résistantes à rude épreuve ; les cyclones d'automne qui balaient Gisborne et Hawke's Bay, en Nouvelle-Zélande déversent en une heure l'équivalent d'un mois de pluie sur des fruits mûrs à point ; les nuages gris et maussades qui s'installent du printemps à l'automne au-dessus des South Downs, en Angleterre condamnent le millésime à la médiocrité. Toute la diversité du climat mondial est là. Les rapports d'étroite dépendance qui unissent les différents climats semblent de plus en plus évidents : un phénomène apparu en un endroit du globe peut déclencher des réactions en chaîne d'un bout à l'autre de la planète.

\section{Le ban des vendanges : une mémoire viticole climatique}

Le droit de ban, ou Bannus, était originellement venu de Germanie et donnait aux souverains des deux premières dynasties, mérovingienne et carolingienne, un pouvoir de commandement théoriquement sans limites. Le ban des

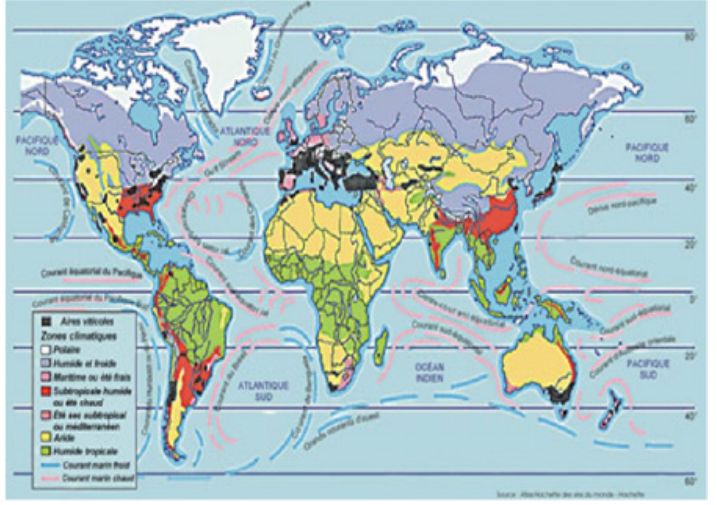

Figure 4. Carte climatique et viticole mondiale (Source: Traitée de viticulture et d'œnologie durables, J ROCHARD, Collection Avenir (Enologie).

vendanges, date du début de la cueillette, établi autrefois par les seigneurs ou les moines, constitue une mémoire des caractéristiques viticoles, et indirectement des conditions climatiques annuelles du passé. Le terme exact est « lever le ban des vendanges », c'est-à-dire l'interdiction de commencer à cueillir le raisin.

En droit féodal, le seigneur haut-justicier disposait du pouvoir d'établir des règlements et des proclamations publiques (droit de ban), sur l'étendue de son domaine. Ainsi, par le ban, le seigneur s'attribuait, dès le XIe siècle, le monopole d'installations telles que four, forge, pressoir, moulin à grain, moulin à huile. Il en résultait, pour les habitants de la seigneurie, l'obligation d'utiliser ces installations, dites banales, contre redevances (souvent en nature). En outre, le seigneur, avec le «banvin» se réservait la possibilité de vendre son vin avant les autres producteurs de la seigneurie [7].

Déjà à l'époque romaine, la date des vendanges n'était pas laissée au libre arbitre du vigneron et reposait sur une délibération publique dans le sens de l'intérêt général et Olivier de Serres soulignait que «ce sont des reliques de l'antique censure de Rome que la police fut le fait des vendanges $\gg[8]$.

Comme le souligne P. VITAL dans son ouvrage « Les vieilles vignes de notre France », « ce serait cependant trop restreindre la portée du ban des vendanges, que de lui attribuer uniquement la facilité de percevoir des redevances. Il avait aussi pour dessein plus noble, la recherche de la qualité, car les experts envoyés dans les vignes pour fixer la date du ban, avaient pour mission essentielle de s'assurer que les raisins avaient atteint la parfaite maturité à l'ouverture du ban. » [9]

J.P. Legrand du CNRS de Paris VI a publié en 1977 et 1978 dans la Revue «La météorologie » une étude visant à cerner les évolutions météorologiques au cours de l'histoire, à partir des dates de vendanges [10,11]. Cette étude s'appuyait sur une sélection, dans la zone Nord et dans le Bordelais, des années particulièrement précoces et tardives. Par exemple pour le Bordelais, de 1753 à 1977, 78 années ont été sélectionnées, 35 précoces et 45 tardives. Une courbe de tendance de variation climatique a été établie à partir de la différence entre le nombre d'années précoces et tardives par intervalle de 50 ans. Pour la période du moyen âge, dépourvue de références sur la date de récolte, l'interprétation était basée sur la disparition progressive du vignoble anglais, J.P. Legrand soulignant 


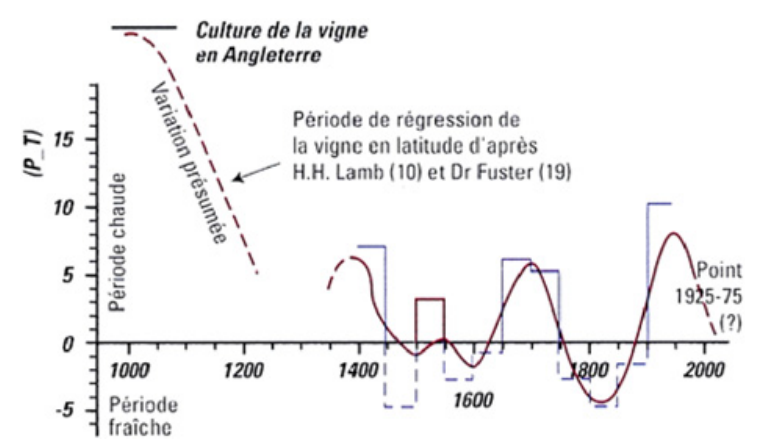

Figure 5. Courbe donnant la tendance des variations climatiques entre 1400 et 1975 . Les variations climatiques naturelles sont indiquées par des tirets.- J.P. Legrand, 1978.

cependant l'importance des aspects politiques dans cette disparition (perte du Duché d'Aquitaine par la France en 1152 au profit des anglais) (Fig. 5).

Cette étude visait à souligner la relation entre les dates d'ouverture des vendanges et le cycle d'activité solaire. Ces observations paraissaient indiquer que les conditions climatiques naturelles pour la fin du $20^{\text {ème }}$ siècle s'achemineraient vers une nouvelle période de refroidissement (sans tenir compte des incidences des activités humaines associées à un réchauffement).

Dans un ouvrage remarquable « Histoire du climat depuis l'an mil », Emmanuel Leroy Ladurie a retracé l'évolution des données climatiques à partir d'une série d'indicateurs, parmi lesquels les dates de vendange des régions septentrionales $[12,13]$. Sa démarche s'appuyait sur des données historiques répertoriées dans différentes régions (Argenteuil, Bourgogne, Champagne, Jura, Suisse). Ces données étaient calculées à partir de la date des bans de vendange en nombre de jour à partir du $1^{\text {er }}$ septembre. E. Leroy Ladurie a souligné la variabilité, au cours du temps des dates de récolte, en liaison avec des modifications climatiques. Ainsi, des récoltes tardives liées au petit âge glaciaire sont intervenues de 1303 à 1859 . Malgré tout, des années précoces ont été observées de manière épisodique, 1636, 1639, 1718, 1719, 1811.

Il est intéressant de confronter cette approche historique à des événements exceptionnels, notamment des éruptions volcaniques importantes qui ont pu conduire par la présence de cendre dans l'atmosphère à une diminution significative de la température, pendant quelques mois, sur une zone importante de notre planète. Benjamin Franklin (1706-1790) a été le premier à souligner que les éruptions volcaniques pouvaient avoir un impact sur le climat avec l'hiver rigoureux de 1783-1784 causé par l'éruption de l'Eldeyjar et du Jökull d'Islande.

Ainsi en 1816, suite à l'explosion du volcan TAMBORA en 1815 , les vendanges ont été très tardives (24 octobre). Ce volcan, situé dans île de SUMBAWA en Indonésie explosa le 10 avril 1815. Son altitude qui dépassait 4000 mètres se réduisit à 2200 mètres après l'éruption.

Un volume de 150 à $175 \mathrm{~km}^{3}$ de pyroclastites (poussières et cendres) fut émis durant 2 à 3 jours jusqu'à $600 \mathrm{~km}$ de distance du volcan et furent projetés dans la stratosphère $(20-30 \mathrm{~km}$ de hauteur) où ils furent transportés par les jets-streams de la haute atmosphère. Pendant plusieurs jours le ciel s'obscurcit sur une surface de la Sicile. En quelques mois les poussières
Table 1. Indice d'Explosivité Volcanique différentes éruptions significatives dans le monde.

\begin{tabular}{|c|c|c|c|}
\hline $\begin{array}{c}\text { Indice } \\
\text { d'Explosivité } \\
\text { Volcanique (I.E.V.) }\end{array}$ & $\begin{array}{c}\text { Volume } \\
\text { des } \\
\text { cendres }\end{array}$ & $\begin{array}{c}\text { Hauteur } \\
\text { du } \\
\text { nuage }\end{array}$ & Exemples \\
\hline 0 & $1000 \mathrm{~m}^{3}$ & $<100 \mathrm{~m}$ & $\begin{array}{c}\text { Kilauea } \\
1959\end{array}$ \\
\hline 1 & $10000 \mathrm{~m}^{3}$ & $100-1000 \mathrm{~m}$ & $\begin{array}{c}\text { Stromboli } \\
2002\end{array}$ \\
\hline 2 & $1000 \mathrm{~m}^{3}$ & $1-5 \mathrm{~km}$ & $\begin{array}{c}\text { Galéras } \\
1992\end{array}$ \\
\hline 3 & $10000000 \mathrm{~m}^{3}$ & $3-15 \mathrm{~km}$ & $\begin{array}{c}\text { Ruiz } \\
1985\end{array}$ \\
\hline 4 & $100000000 \mathrm{~m}^{3}$ & $10-25 \mathrm{~km}$ & $\begin{array}{c}\text { Galunggung } \\
1982\end{array}$ \\
\hline 5 & $1 \mathrm{~km}^{3}$ & $>25 \mathrm{~km}$ & $\begin{array}{c}\text { Mt St Helens } \\
1981\end{array}$ \\
\hline 6 & $10 \mathrm{~km}^{3}$ & $>25 \mathrm{~km}$ & $\begin{array}{c}\text { Krakatau } \\
1883\end{array}$ \\
\hline 7 & $100 \mathrm{~km}^{3}$ & $>25 \mathrm{~km}$ & $\begin{array}{c}\text { Tambora } \\
1815\end{array}$ \\
\hline 8 & $1000 \mathrm{~km}^{3}$ & $>25 \mathrm{~km}$ & $\begin{array}{c}\text { Yellowstone } \\
2 \text { Ma. Il y a } \\
\text { environ } \\
640 \text { 000 ans }\end{array}$ \\
\hline
\end{tabular}

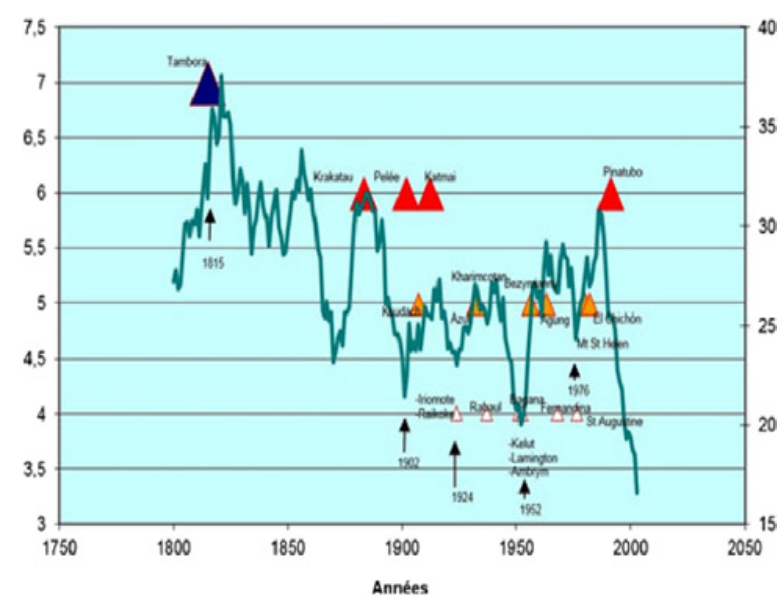

Figure 6. Evolution des dates des vendanges (en moyenne mobile sur 10 ans), données d'E. Leroy Ladurie et éruptions volcaniques.

et aérosols se répandirent dans l'atmosphère provoquant des modifications climatiques pendant plusieurs années. Son effet sur le climat a semble-t-il été très significatif : pas d'été en Nouvelle Angleterre et dans l'Europe de l'Ouest, température inférieure de 3 degrés par rapport à la normale, gel et neige tous les mois dans certaines régions européennes.

Les volcans indonésiens KRAKATOA (1883) et Augun (1963), le Mont Saint Helens (1980) aux EtatsUnis, El chichon (1982) au Mexique, et le Pinatubo (1991) aux Philippines ont également contribué à un refroidissement significatif.

Les principales éruptions volcaniques sont répertoriées dans le Tableau 1 (Indice d'explosivité des éruptions).

La Fig. 6 répertorie (moyenne mobile sur 10 ans) les données historiques des vendanges établies par E. Leroy Ladurie et les principales éruptions volcaniques des $19 \mathrm{e}$ et 20e siècles. 


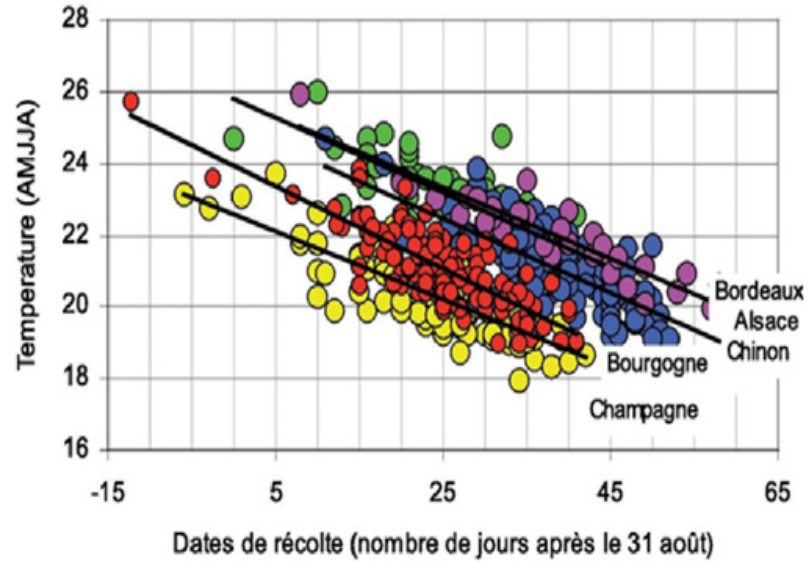

Figure 7. Corrélation entre la température et des dates de vendanges de différents vignobles (Source V. Daux /CNRS).

Les données historiques récentes permettent d'appréhender de manière simple l'influence du changement climatique sur les dates de vendanges (Fig. 7). A partir d'une date de vendange moyenne actuelle, il suffit de retrancher un nombre de jours correspondant à l'élévation de température prévue divisée par 10 [14]. Par exemple une élévation moyenne de température de $1^{\circ} \mathrm{C}$ des températures maximales de la période de croissance (avril à août) correspond à une avancée de la date d'ouverture de vendange moyenne de 10 jours (8 à 12 jours).

\section{Conclusion}

La prospective est un exercice difficile, soumis à de nombreuses incertitudes, en particulier pour le climat, influencé par des facteurs interdépendants, ce qui justifie la prudence dans l'interprétation des évolutions. Néanmoins, les changements et notamment le réchauffement semblent inéluctables. Des études ont permis de cerner les évolutions des dates de vendange actuelles. Elles complètent les travaux des historiens qui ont étudié la variabilité du ban de vendange au cours des cinq derniers siècles. La précocité observée depuis la fin des années 1980 est de 10 à 20 jours comparativement au milieu du XXe siècle [15].

Au regard des évolutions actuelles prévues depuis une quinzaine d'années, pour une évolution de l'ordre de $0.5^{\circ} \mathrm{C}$, il parait plausible d'imaginer des changements significatifs concernant les itinéraires viticoles et œnologiques au cours des prochaines décennies et probablement à terme, une modification des choix variétaux.

Parallèlement à l'élévation moyenne de la température, il semble qu'une modification du climat puisse accentuer les effets extrêmes. Périodes sèches prolongées, fortes pluies « tropicales », tempêtes violentes sont autant de phénomènes susceptibles de modifier les conditions culturales. Vis à vis de la gestion du sol, une telle hypothèse justifierait à la fois une généralisation des moyens de lutte contre le ruissellement et l'érosion, et une gestion optimale de l'eau en conditions estivales. Cette évolution pourrait conduire à une adaptation des pratiques traditionnelles locales (labour, enherbement, mulch) et

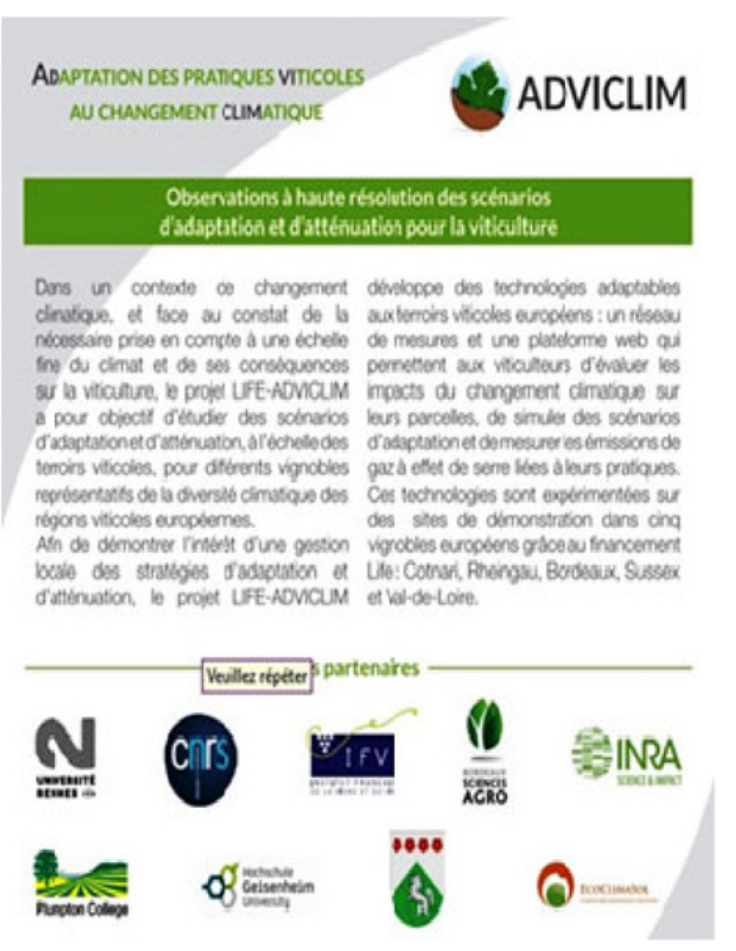

Figure 8. Site internet du projet ADVICLIM www . adviclim.eu

poserait dans certaines régions avec plus d'acuité la nécessité d'irriguer.

La mise en place d'observatoires viticoles régionaux dans le cadre d'un réseau international peut permettre aux viticulteurs de faciliter l'adaptation progressive de leurs vignobles aux changements climatiques. Dans ce cadre, le projet européen LIFE ADVICLIM www.adviclim.eu a pour objectif d'étudier des scenarios d'adaptation et d'atténuation, à l'échelle des terroirs viticoles, pour différents vignobles représentatifs de la diversité climatique des régions viticoles européennes. Le projet comporte également une plate-forme Web qui permet aux viticulteurs d'évaluer les impacts du changement climatique sur leurs parcelles (Fig. 8).

En complément, il est possible d'imaginer que les observations viticoles recueillies, mises en réseau, permettent d'alimenter une base de données mondiale d'indicateurs biologiques, complétant les scénarios climatiques établis par le GIEC.

\section{Références}

[1] J. Rochard, La vigne : une plante bio indicatrice emblématique des variations climatiques, revue française d'œnologie, 273 (2015)

[2] J. Rochard, Traité de viticulture et d'œnologie durables, éditions Avenir œnologie (2003)

[3] J. Rochard, Changements climatiques, perspectives pour la viticulture, Revue des œnologues, 110 (2004)

[4] A. Carbonneau, C. Riou, D. Guyon, J. Riom, Agrométéorologie de la vigne en France, Commission des Communautés Européennes (1992)

[5] P. Huglin, C. Schneider Biologie et écologie de la vigne, Lavoisier, Paris (1998)

[6] O. Clarke, Atlas Hachette des vins du Monde, Ed. Hachette, Paris (1995) 
[7] H.Chobaud, Le ban des vendanges et la date du début des vendanges dans le Comtat Venaissin du XIVe au XIXe siècle, Annales de la Société d'Ethnologie française, p. 55-60 (1950)

[8] A. Guerreau, Climat et vendanges (XIVeXIXe siècles) : révisions et compléments, in Histoire et Mesures, X 1-2, p. 89-147 en ligne (1995)

[9] P. Vital, Les vieilles vignes de notre France, réédition Wallada (1984)

[10] JP. Legrand, Fluctuations météorologiques, vendanges et activité solaire, La météorologie, 1ère partie VI, 9, 73p (1977)
[11] JP Legrand, Fluctuations météorologiques, vendanges et activité solaire, La météorologie VI, 12 (1978)

[12] E. Le Roy Ladurie, Histoire humaine et comparée du climat en Occident. Canicules et glaciers XIIIeXVIIIe siècles, Paris, Fayard (2004)

[13] E. Le Roy Ladurie, Disettes et révolutions, 1740 1860, Paris, Fayard (2006)

[14] V. Daux, La reconstruction climatique à partir des dates de vendanges, Revue de la BNF 2010/3, $n^{\circ} 36$, p. 26-33

[15] J. Rochard, J.R. Clement, A. Srhiyeri, Zonage et modification du climat : Évolution des dates de vendange, ITV France, Pôle environnement en ligne 